\title{
The Integrated Intelligent Operation and Maintenance Management Solution for Cloud Data Center in the Aerospace Domain
}

\author{
Hongyan Chen, Hongwei Qi, Hang Yin
}

\author{
Beijing Institute of Tracking and Telecommunications Technology, Beijing 100094, China \\ child_smile@163.com
}

\begin{abstract}
The cloud data center for aerospace trial mission must be high-performance, high stability, high security and scalable computer network architecture, in order to support the need for continuous updating, upgrading and development in the future, and ensure the smooth implementation of the aerospace test task. According to Characteristics and technical requirements of IT operation and maintenance for cloud data center of Aerospace test system, With the domestic self-controlled hardware and software as the core, Adopt subsystem, hierarchical, modular design concept of system architecture, Building an all open, component-based architecture prototype, Integration of resource monitoring, automation operation and maintenance, operation and maintenance process management, Build a reliable, scalable and high-performance solution for integrated IT operation and maintenance management. Escorting the stable operation of the aerospace trial mission system.
\end{abstract}

Keywords: Aerospace test, Intelligent operation and maintenance, Integration model, Independent controllable.

\section{Introduction}

With the rapid development of the information, network and digitalization of the society, the construction of the network information center, cloud data center and cloud computing center of the aerospace test task system is also expanding. With the rapid development of China's aerospace industry, the IT information system of the aerospace test mission system is becoming more and more large. Because of the complex and changeable environment of IT network management, such as multi system, multi service and multi-vendor equipment, the difficulty of IT maintenance becomes geometric multiplier, and the risk and hidden danger of IT information technology are also increasing. At present, the IT system has been providing services for the core business, basic business, daily office and other aspects of the aerospace test task system. The aerospace test task is becoming more and more dependent on the IT system, which puts forward a higher demand for the stable and efficient operation of the whole system. How to ensure the stable and safe operation of the whole IT system in the aerospace test task system has gradually become a problem that the management and system engineers of the aerospace business pay more attention to.

Cloud data center is the core of providing cloud computing services and upgrading of traditional data centers. Whether it is a traditional data center or a cloud data center, from their life cycle, operation and maintenance management is the longest period in the whole life cycle. In the operation and maintenance management of the traditional data center, the operation and maintenance management of the aerospace service system cloud data center is relatively passive and lagged because there is no advanced IT operation and maintenance management system. When the system is in serious trouble, it can detect the exception, which results in the slow processing of the fault and sometimes even affects the normal operation of the aerospace test mission system. Therefore, timely and accurate understanding of the performance of equipment, utilization of resources, and operation bottleneck of the business system has an indispensable reference value for the information construction of the aerospace test task system.

In summary, how to effectively integrate the system resources in the aerospace test task system, to maximize the potential of the IT system in the cloud data center, to maximize the operation and maintenance efficiency and enhance the security of the system, has become an urgent problem to be solved in the aerospace test task management, and a set of advanced and safe functions can be built. 
It is imperative to rely on the integrated monitoring and control system of IT operation and maintenance.

\section{Status and Problems}

With the increasingly heavy task of aerospace research and testing, with the continuous deepening of the aerospace system information construction, the construction of the cloud data center network is gradually mature, and the degree of data concentration is becoming more and more high. At present, the present situation and main problems of IT system for aerospace research and test tasks are as follows:

All kinds of core business and basic applications in the aerospace trial mission system are expanding, and they are more and more dependent on the IT network system. The lack of a set of unified monitoring system for IT system software and hardware, cannot timely understand the IT system software and hardware equipment running trend, cannot quickly fault location, the failure processing efficiency is low;

The scale of aerospace test business and application is increasing. The division of planning, security, management and maintenance is more and more meticulous. It lacks the monitoring of the health status and running trend of the IT system. It is unable to determine whether the business system has the bottleneck of operation, whether it needs expansion or network optimization;

It is impossible to centrally manage the operation information and alarm information of various equipment and business systems, and carry out intelligent analysis and statistics on the above information, and get the data that is beneficial to the management and maintenance of the network and achieve the purpose of efficient and quick processing;

Lack of data center computer room diversification of assets visual reproduction ability, is not conducive to the operation and maintenance personnel of the computer room resources control;

Facing the complex aerospace trial IT environment, there is no standardized and automated operation and maintenance management process, and lack of perfect fault handling and quick repair mechanism.

\section{Requirement Analysis}

Through in-depth understanding of the status quo of cloud data center informatization and operation and maintenance management of the aerospace test mission system. It's operation and maintenance service need to change the traditional operation and maintenance management mode, change passive mode into active operation and maintenance, and achieve $7 * 24$ hours continuous operation and maintenance, and then ensure the normal operation of the aerospace test task system. Therefore, the specific requirements for the operation and maintenance management platform of the cloud data center in the aerospace trial mission system are as follows:

It can monitor and control the operation of all the business resources in a comprehensive and detailed way, and provide an integrated system management mode. It can dynamically monitor the performance of network and business resources in real time, and can objectively evaluate the current system health status and long-term system performance changes and trends through cloud computing and large data analysis, and provide scientific basis for system upgrading, expansion and development;

With comprehensive and in-depth data statistics, analysis and management functions to ensure the security, reliability and high performance of the large data system, so as to form the best backstage support system for large data analysis and processing;

Providing all kinds of applications and business functions needed in the IT system of enterprise level cloud data center, and integrated, integrated and intelligent management mode can reduce the cost of system operation and maintenance management;

Realize business 3D visual management, analyze deep-seated causes and other issues. 


\section{Solution}

\subsection{System Technology Architecture}

The integrated intelligent operation and maintenance system of the aerospace test mission system cloud data center is divided into four levels, namely, the data display layer, the operation and maintenance management layer, the data processing layer and the data acquisition layer. The platform is modular design, and modules are loosely coupled. The new module can be directly connected to the platform, and modules communicate through interfaces and message queues. The four layer structure is based on the operation and maintenance portal, the IT service management platform, the integrated monitoring platform, the CMDB, the server monitoring platform, the IT resources and the business system, and embodies the design concept of the integrated operation and maintenance of "supervision and control". The technical architecture is shown in Figure 1:
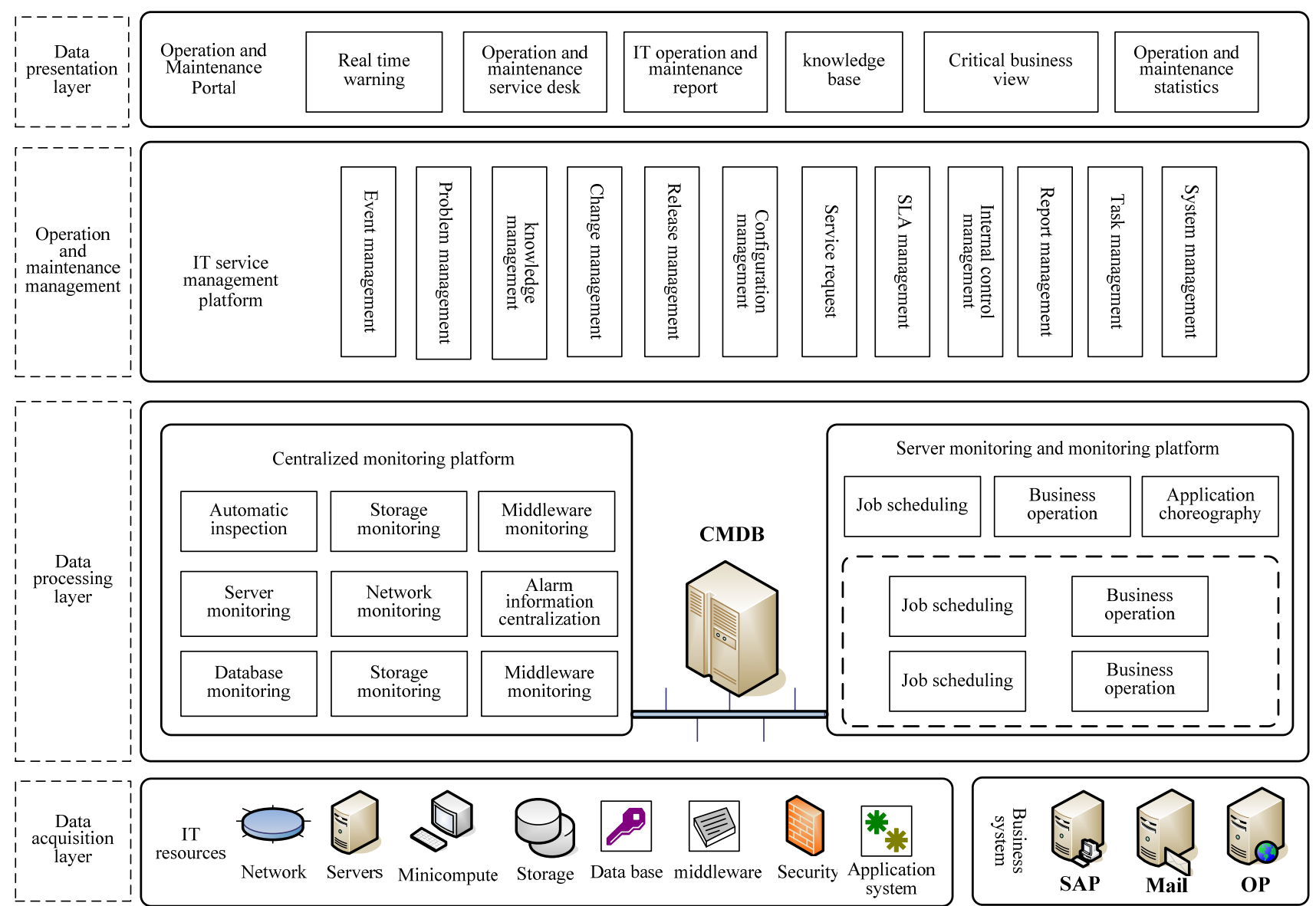

Fig 1. System technology architecture

\subsubsection{System Technology Architecture}

The data acquisition layer is the foundation of the whole management platform, which is responsible for collecting the data needed for the platform operation. The data acquisition layer, through a variety of network protocols, including SNMP, SSH, TELNET, PING, JDBC, JMX, SMI$\mathrm{S}$, etc., obtains the required index information from the managed equipment, and puts the collected data in the cache for analysis and operation, and then stores it into the database for the upper platform to analyze and display. The platform is built with an extensible resource capability library model. For the dissatisfied manufacturers, models and indicators, the system can be configured through the system without two times of development. It supports a custom extension of the monitoring index through SNMP, JDBC, and JMX.

\subsubsection{Operation and Maintenance Management Layer And Data Processing Layer}

Operation and maintenance management layer and data processing layer include IT service management platform, CMDB, centralized monitoring platform, server monitoring platform and 
other cloud platform subsystems. All systems use domestic hardware and own software copyright software, and have complete independent intellectual property rights. Each subsystem in the cloud platform works independently and data linkage. It has good compatibility and scalability.

The IT service management platform manages the IT resources and environment comprehensively through the functions of system management, configuration management, event management, problem management and knowledge management. It provides the management and statistics of large data and its display pages to meet the needs of the daily work of the users.

The centralized monitoring platform realizes the operation status monitoring, fault management and performance management of IT infrastructure and business systems. It has the ability to integrate with other platforms such as cloud platform, power environment system and other platforms, and provides a variety of data integration methods, and can use large data platform to construct the evaluation and analysis model of operation and maintenance index, according to the law of historical data change, mining related information such as business, index, failure and so on, helping the operation and maintenance personnel find the improvement of the problem root. The platform integrates monitoring information, alarm information presentation, business association analysis and alarm correlation analysis.

CMDB implements the configuration information management of all IT resources in the data center, ensures the integrity and precision of the configuration items in the data center, constructs the operational and maintenance management metadata, and provides resource data and large data analysis for the monitoring and operation process.

The server monitoring platform integrates the discrete monitoring task and the operation control task into an automated process with a certain management goal and business goal through an automated process development tool, and realizes the automation of the process driven operation and maintenance. By scheduling and integrating a large number of dispersed agents and adapters, the service automation components centrally distributed the decentralized IT infrastructure and business system management to a unified management platform. Integrated system monitoring and service automation have laid the foundation for centralization and automation of management.

\subsubsection{Data Presentation Layer}

The uppermost layer is the display layer of the $\mathrm{B} / \mathrm{S}$ architecture, which is the unified portal of the whole service management system, through the unified user authentication and single sign on. Through the visualization of the data center $3 \mathrm{D}$, the business and equipment information is presented from the angle of three-dimensional visualization, which includes the visualization of the computer room environment, the visualization of assets, the visualization of the wiring, the visualization of the capacity and the visualization, and the requirement of the diversified monitoring and management, and the accurate mastery of the IT by the operation and maintenance management personnel. Operation situation and service level of operation and maintenance.

\subsection{System Functional Architecture}

The integrated intelligent operation and maintenance management system functional architecture, as shown in Figure 2, uses multi-layer architecture and modular design patterns, including operation and maintenance monitoring management, operation and maintenance process management, portal, operation and maintenance automation management, operation and maintenance of large data analysis and other subsystems. The whole system platform uses J2EE architecture, fully graphical $\mathrm{B} / \mathrm{S}$ mode, and can be more portability, and can be deployed across platform based on different operating systems (Windows, Linux, homemade unicorn, etc.). A unified and open, component-based monitoring and management platform supports a variety of databases (MySql, Oracle, domestic databases, etc.), supporting domestic middleware such as TongWeb, supporting OpenJDK, and providing a third party system integration interface that conforms to the national information technology service standards (ITSS). 


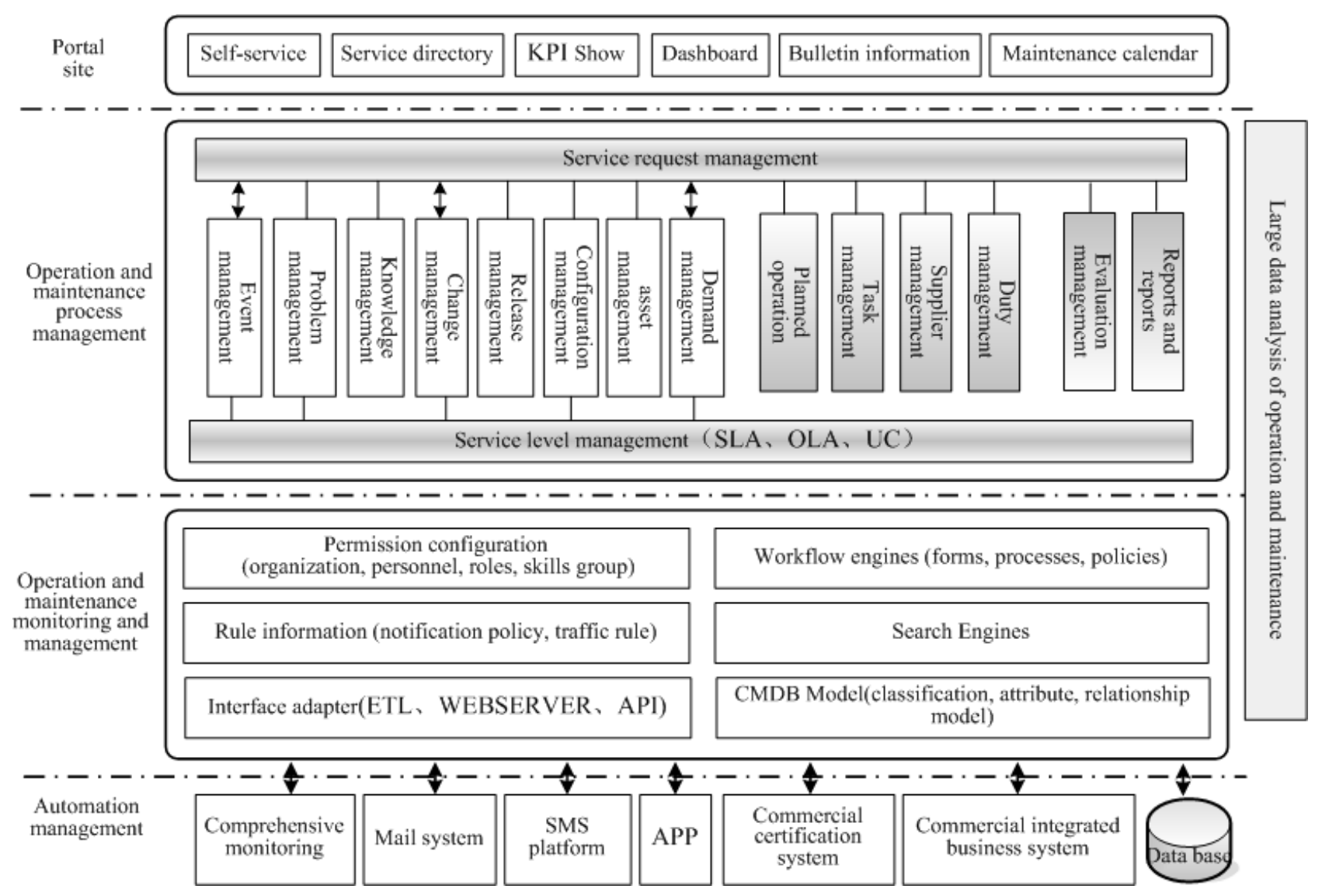

Fig 2.System functional architecture

The integrated intelligent operation and maintenance management system is divided into Portal service layer, DHS (information processing) service layer and DCS (Information Collection) service layer. Each layer can be deployed on the same or different host on the basis of the actual situation of the customer's IT environment. According to the customer's management object scale, the management capacity planning is carried out by single or multiple DCS. Through centralized or distributed deployment, the flexible management of IT resources in the complex structure of aerospace test system, external network, headquarters / branch, etc. can be realized.

Unified operation and maintenance portal is a unified access, unified authentication and custom home page working interface of integrated intelligent operation and maintenance management system of cloud data center. It supports the integration of the three party system, such as cloud computing platform and mobile ring monitoring system, so as to realize the centralized display of the third party system and the unified push of the alarm. Through the unified authentication function, users can manage the user accounts of other systems on a single interface, modify and configure the privileges of different roles, and add user accounts.

Through modular design concept, it provides operation and maintenance Mobile APP, supports Android, IOS operating system, supports notification bulletin release, alarm notification, work order submission and processing, configuration data query, knowledge base query and mobile inspection, so that IT operators are no longer restricted by region and can be mobile or wireless anywhere. The network is connected to the platform for operation and maintenance.

The full open and component-based architecture is used to reflect the resource allocation of various devices in the user network system and the setting of important parameters, to automatically search the related configuration information of all the equipment in the network system, and to identify the hardware configuration information of the type, model, manufacturer and interface of the equipment. A flexible threshold setting is used to measure the network usage and reflect the health of resources. Through the interface between the alarm management module and the operation and maintenance process management module, the operation and maintenance process management module is 
forwarded to the operation and maintenance process management module after the designated event, and the event processing unit is automatically initiated and the alarm failure is solved in time.

\section{Summary}

If you follow the "checklist" your paper will conform to the requirements of the publisher and facilitate a problem-free publication process.

\section{References}

[1]. Network operation and maintenance management system [DB/OL]. http://www.docin.com.

[2]. Jianping LIU, Longjiang YANG. An analysis of the integrated mode of operation and maintenance management [J]. software engineer,2012(06):37-39.

[3]. Weijian TAN. Construction of centralized accounting and settlement monitoring system [J]. Communication technology in Guangdong,2005(04):10-13+43.

[4]. Hongyan CHEN, Junwei WAN, Hongwei QI. Research on cloud smart office integrated management system solution based on Internet of things [J/OL]. Modern electronic technology,2018(10):85-89[2018-05-30].https://doi.org/10.16652/j.issn.1004373x.2018.10.022.

[5]. Hongyan CHEN, Junwei WAN. Research and application of enterprise website performance optimization based on Web [J]. Internet of things technology,2018,8(02):67-69+73.

[6]. Junwei WAN, Hongyan CHEN, Jing ZHAO. Application verification of cloud computing technology in real time aerospace test mission area [J]. Netinfo Security,2017(05):63-68.

[7]. Junwei WAN, Hui ZHAO etc. The development status and application analysis of autonomous controllable information technology [J]. Journal of Spacecraft TT\&C Technology, 2015, 34 (04):318-324.

[8]. Hui ZHAO, Junwei WAN etc. Application Research of autonomous control technology in experimental task domain [J]. Journal of Spacecraft TT\&C Technology,2015,34(02):109-114.

[9]. Hongyan CHEN, Junwei WAN, Qi WANG. Design and implementation of large data high performance sorting algorithm [J]. Journal of Spacecraft TT\&C Technology,2015,34(02):120127. 